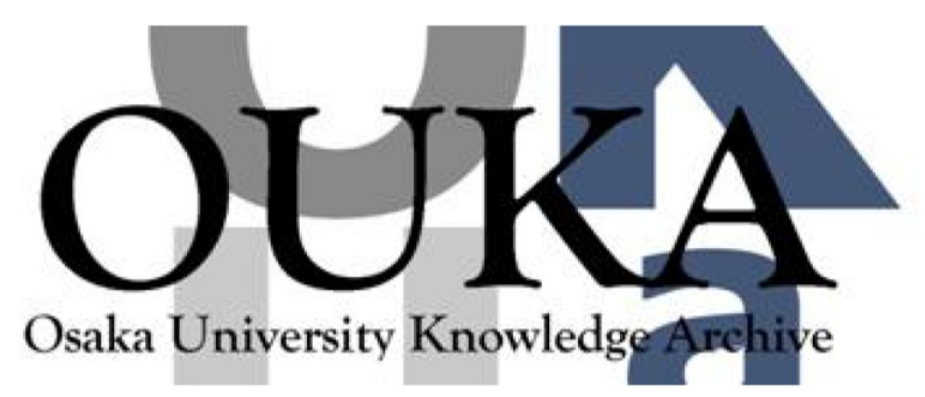

\begin{tabular}{|c|l|}
\hline Title & $\begin{array}{l}\text { Phase measurement interferometric microscopy of } \\
\text { stacked fishnet metamaterials }\end{array}$ \\
\hline Author(s) & Matsui, T.; Miura, A.; Nomura, T. et al. \\
\hline Citation & $\begin{array}{l}\text { Proceedings of SPIE - The International Society } \\
\text { for Optical Eng ineering. 8269 p. 82692P }\end{array}$ \\
\hline Issue Date & $2012-02-22$ \\
\hline oaire:version & VoR \\
\hline URL & https://hdl. handle. net/11094/76948 \\
\hline rights & \\
\hline Note & \\
\hline
\end{tabular}

Osaka University Knowledge Archive : OUKA

https://ir. Library. osaka-u. ac. jp/

0saka University 


\title{
Phase measurement interferometric microscopy of stacked fishnet metamaterials
}

\author{
T. Matsui ${ }^{* a}$, A. Miura ${ }^{a}$, T. Nomura ${ }^{a}$, H. Fujikawa ${ }^{a}$, K. Sato ${ }^{a}$, N. Ikeda ${ }^{b}$, D. Tsuya ${ }^{b}$, M. Ochiai ${ }^{b}$, \\ Y. Sugimoto ${ }^{b}$, H. T. Miyazaki ${ }^{b}$, M. Ozaki ${ }^{c}$, M. Hangyo ${ }^{c}$, and K. Asakawa ${ }^{d}$ \\ ${ }^{a}$ Toyota Central R\&D Labs. Inc., 41-1, Yokomichi, Nagakute, Aichi, 480-1192, Japan; \\ bational Institute for Materials Science, 1-2-1, Sengen, Tsukuba, Ibaraki, 305-0047, Japan; \\ 'Osaka University, 2-1 Yamadaoka, Suita, Osaka 565-0871, Japan: \\ ${ }^{\mathrm{d}}$ University of Tsukuba, Tsukuba, Ibaraki, 305-0006, Japan
}

\begin{abstract}
Alternating stacks of metal and dielectric films with nano-hole arrays, called fishnet structures, control the propagation of electromagnetic waves. In such a structure, changing a dimension or a shape, especially the change in shape of nanoholes, affect propagation constants. In this study, we report the dispersivity of fishnet structures is controllable with different hole shapes, by measuring the interferometric fringe in various wavelengths. Two structures were fabricated, which consist of five alternating stacks of aluminum and silicon dioxide with nano-hole arrays. The holes in one of the structures are circular with diameters of $500 \mathrm{~nm}$, and the other are square with $500 \mathrm{~nm}$ sides. The lattice constant in each case is $1,000 \mathrm{~nm}$. Since fishnet structures are wavelength-dependent structures, the variable-wavelength interferometric microscope was set up. The phase shift of the circular hole and the square hole fishnet were about 110 degrees and 85 degrees, respectively, within a tunable wavelength from $1,470 \mathrm{~nm}$ to $1,545 \mathrm{~nm}$. These values were equivalent to a refractive-index-change of 0.8 and 0.6 , respectively. From these results, fishnet structures indicate high dispersivity within target wavelengths. The dispersion of fishnet structure can be controlled by the shape of the hole.
\end{abstract}

Keywords: plasmon, fishnet, metamaterial, interferometric, microscope

\section{INTRODUCTION}

Sub-wavelength periodic structures such as dielectric gratings or metallic sub-wavelength apertures enable control of wave propagation by changing their structural parameters, such as the periodicity, the width of the slits, or the radii of the holes [1-3]. Previously, we reported experiments of RGB color filters which consist of periodic arrays of subwavelength holes on optically thick aluminum films [4]. The transmittance band of the filters was controlled only with slight changes in the periodicity and the shape of the holes. The fabrication process of this structure is relatively simple since it has only one metallic layer to be etched. Nevertheless, it is difficult to simultaneously control multiple optical characteristics, e.g. dispersion relationships, with such simple structures.

Recently, thanks to improvement of nano-fabrication techniques, more complex structures with multiple layers can be fabricated, even with combinations of metals and dielectrics. The fabrication of the fihnet structure, that is, multiple layers of metal and dielectric with an array of sub-wavelength holes perforated through all layers, is an important challenge in nano-fabrication to realize optical metamaterials (MM) to control optical characteristics by their structures[5,6]. With fishnet structures, slight changes in the structures, especially the shape of the sub-wavelength holes, give rise to a drastic change of optical properties [7]. Such structures have a potential to improve small optical elements, such as planar lenses or planar prisms by designing the shape and the layout of the nano-holes or nano-slits [8, 9].

In order to evaluate fishnet structures, transmission phase of light in a small specific area is a point of our interests. However, up to now, optical metamaterials have been evaluated by measurement of transmittance and reflectance spectroscopy. The optical properties have been discussed indirectly by comparing numerically calculated spectra and an experimental one $[10,11]$.

Here, in order to evaluate fishnet metamaterial structure, an interferometric microscopy is chosen in this study. By using this, it is possible to obtain an image of interferometric fringes of a specific area, which reveals the distribution of phase

Photonic and Phononic Properties of Engineered Nanostructures II edited by Ali Adibi, Shawn-Yu Lin, Axel Scherer, Proc. of SPIE Vol. 8269, 82692P · C 2012 SPIE - CCC code: 0277-786X/12/\$18 - doi: 10.1117/12.907248 
differences on the normal microscopic image, and we experimentally demonstrate the dispersion control of fishnet structures with different hole shapes

\section{EXPERIMENTAL}

The fishnet structure was fabricated using multilayer deposition, electron beam lithography, and layer by layer dry etching (Fig. 1). First, alternating stacks of six silicon dioxide $\left(\mathrm{SiO}_{2}\right)$ layers, $80 \mathrm{~nm}$ thick, with five aluminum (Al) layers, $20 \mathrm{~nm}$ thick, were deposited on the fused silica substrate by magnetron sputtering. Total thickness is $580 \mathrm{~nm}$. Secondly, ZEP-520A electron beam resist was spun on the film stack. Sub-wavelength hole patterns were defined using an electron beam writer (Elionix, ELS-7000). Finally, holes were perforated by dry etching using the resist pattern, layer by layer. The reactive ion etching and the inductively coupled plasma (ICP) etching is used for etching the $\mathrm{SiO}_{2}$ layers and the $\mathrm{Al}$ layers, respectively. Unit cell of the each fishnet MMs have the lattice constant of 1,000nm. Hole-shape of fishnet MMs are circular with diameters of $500 \mathrm{~nm}$, and square with $500 \mathrm{~nm}$ sides against circular fishnet MMs and square fishnet MMs, respectively. Note that, to compensate error in dimension, several samples are fabricated by scaling entire lithography patterns.

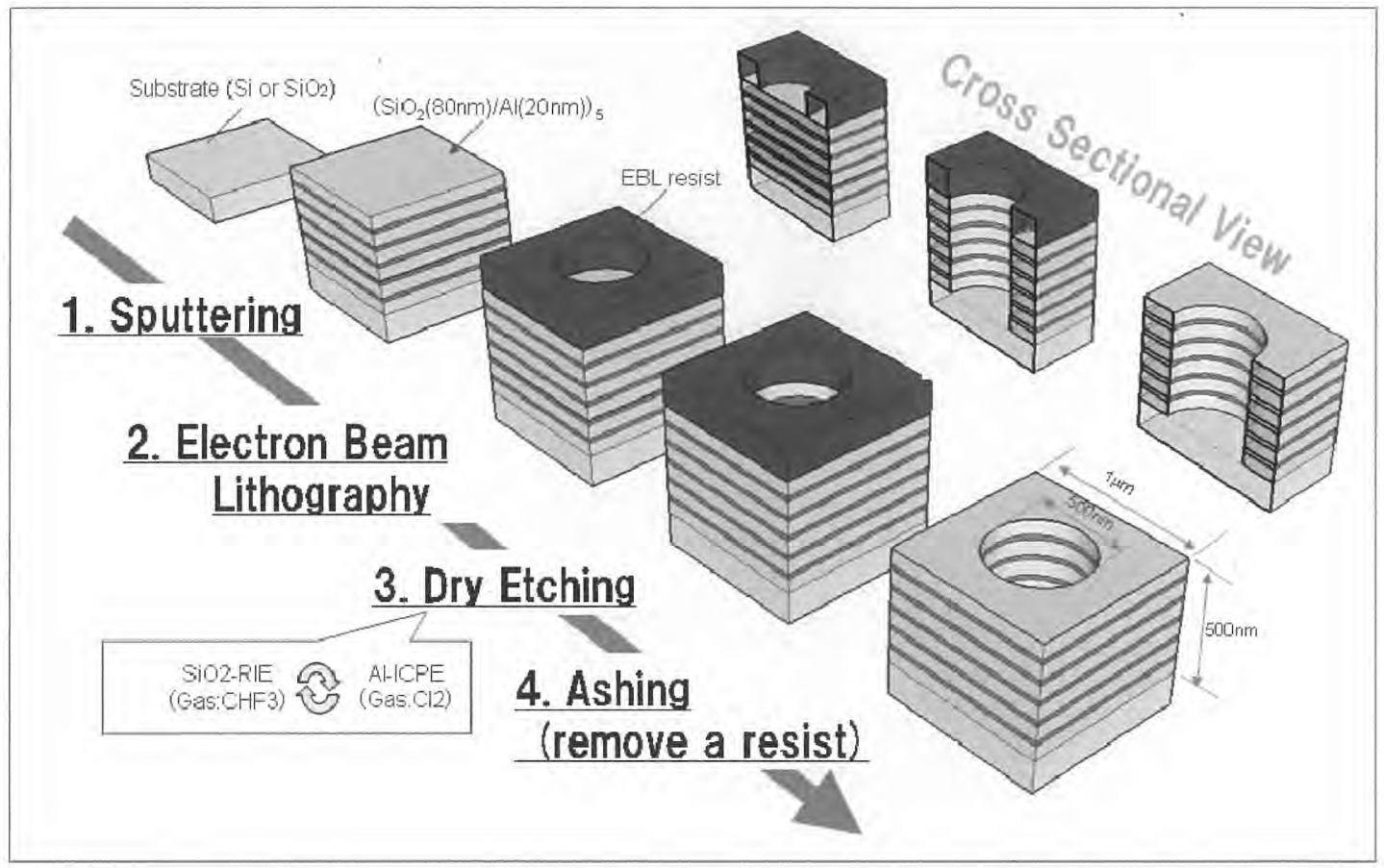

Fig. 1. Fabrication Process.

In order to capture wavelength dependent characteristics of the fishnet structure, a newly designed interferometric microscope system was set up. This microscope system consists of a Mach-Zehnder interferometer integrated with a microscope (Olympus, BX-51IR) and an external-cavity wavelength-tunable diode laser (New Focus velocity TLB-6326, $\Delta v<300 \mathrm{kHz}, \lambda=1,470-1,545 \mathrm{~nm})$.

There are four criteria for an interferometric microscope system to produce interferometric images for phase difference analysis. The first one is that, obviously, the polarization direction between the sample and the reference must be matched [12]. This is satisfied by careful layout of the mirrors and half wave plates. Second, the optical path length of the sample arm and reference arm must be matched. More precisely, the difference of the path length of two arms must be smaller than the coherence length $\Delta L$ to make interference occur. Here $\Delta L$ is expressed as $\Delta L=c_{0} d \Delta v$ where $c_{0}$ is the velocity of the light. In our case, the coherence length is longer than $1,000 \mathrm{~m}$. This is far longer than the path length of two arms; therefore, this criterion is naturally satisfied in the laboratory experiment. The third criterion is that all of the 
optical elements in the setup must be achromatic in target wavelength range. A thin pellicle beam splitter is adapted as an element for both divining and combining the beam to keep system achromatic by minimizing wavelength dispersion. The fourth criterion is that the wavefront-curvatures of the two beams have to be matched. In this experiment, since the phase difference will be measured using the interferometric fringe pattern, the flat fringe pattern is desired. That is, the fringes should be straight lines and distance of each fringe line is proportional to the phase difference. However, if two wavefront-curvatures are not matched, the shape of the interferometric fringes will be coaxial circles, namely "Bull's eye" pattern [13] (Fig. 2(a)). On the other hand, when the wavefront-curvatures of the two beams exactly matched, the fringe pattern will be flat (Fig. 2(b)). It is achieved by tuning the wavefront-curvatures via arrangement of the lenses. This is intuitively grasped as follows: a parallel light and focused light have an infinite and minimum wavefront curvature, respectively. Thus, by tuning the focus of the both beams, the two wavefront-curvatures are changed and as the result, the fringe pattern is controlled. Therefore, the system should have ability to control the lens arrangement for both beams precisely. Note that, the wavefront curvature radii are easily estimated from Gaussian beam relations and the $\mathrm{ABCD}$ law [14].

(a)

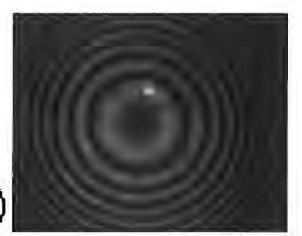

Fig. 2. Interferometric fringe pattern (a) Bull's eye pattern, (b) Flat pattern.

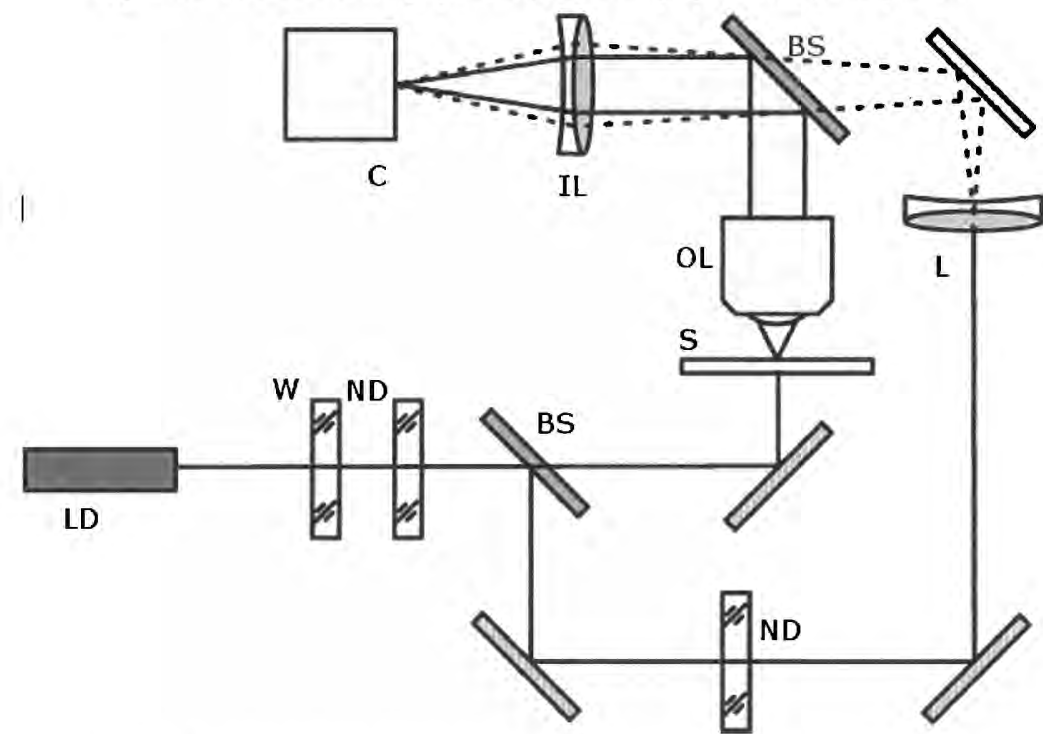

Fig. 3. Experimental setup for wavelength-tunable interferometric microscope. LD, external-cavity wavelengthtunable diode laser $(\lambda=1,470 \sim 1,545 \mathrm{~nm})$; $\mathrm{W}$, achromatic half-wave plate; ND, neutral density filter; BS, pellicle beam splitter; S, sample; OL, objective lens (Olympus LMPlanIR, 20X, NA=0.40); L, achromatic doublet lens

$(\mathrm{f}=40.0 \mathrm{~mm})$; IL, imaging lens $(\mathrm{f}=180.0 \mathrm{~mm}) ; \mathrm{C}$, near infrared camera(Hamamatsu photonics, $\mathrm{C} 10633-13$,

$$
\lambda=900 \sim 1700 \mathrm{~nm}) \text {. }
$$

The optical setup we built is depicted in Fig. 3. The path of the light is as follows. First, the polarization of the beam is rotated by achromatic half-wave plate, and then the beam is divided into sample path and reference path by a pellicle beam splitter. The sample beam passes through a sample and the objective lens. The reference beam passes through a neutral density filter and an achromatic doublet lens. The wavefront curvature of the reference beam is matched with the sample beam by moving the achromatic doublet lens. After the sample beam and the reference beam are recombined by the pellicle beam splitter, the combined beam passes through an imaging lens, and finally it forms interferometric images onto the near infrared camera. 


\section{RESULTS AND DISCUSSION}

Figure 4 shows the top and cross-sectional view SEM images of fabricated fishnet MMs. From cross-sectional SEM image, sidewall angle arisen from fabrication process was observed. The value of sidewall angle was about 80 degrees.
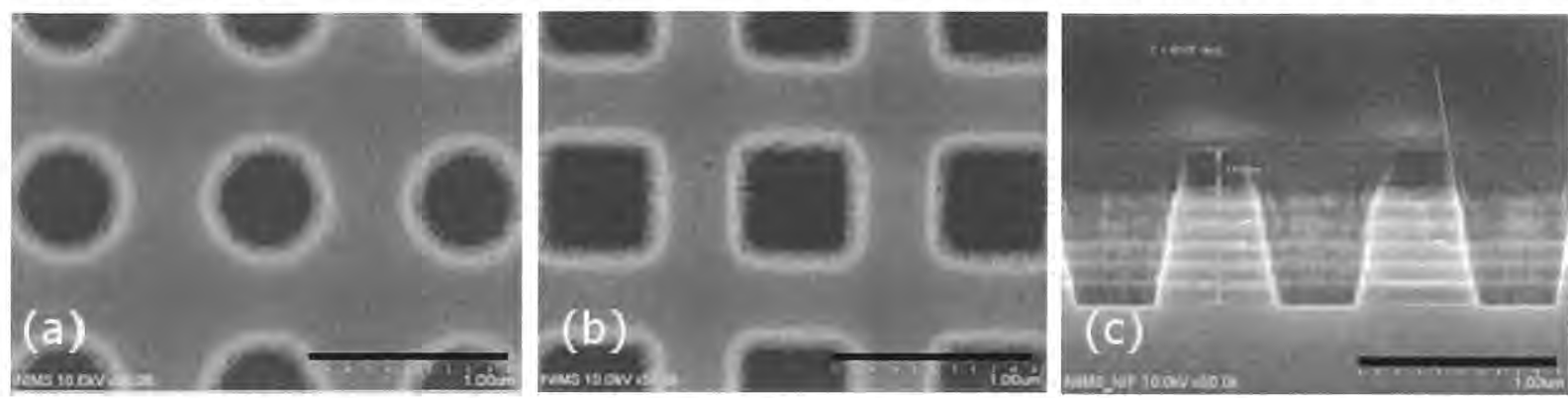

Fig. 4. Scanning electron micrograph of a fabricated fishnet structures (a) top view image of circular hole fishnet, (b) top view image of square hole fishnet, (c) cross sectional view. Scale bars are $1 \mu \mathrm{m}$

For interferometric observation, a bare substrate region which was partially-overlapped with the fishnet structure was additionally milled by using a focused ion beam (Fig. 5(b)). Since etching rate of the fishnet region was faster than that of multilayer $\left(\mathrm{Al} / \mathrm{SiO}_{2}\right)$ region, structure result in Fig. 5(c). After this additional process, three regions which are bare substrate S1, etched substrate S2, and fishnet MMs S3 were obtained.

(a)

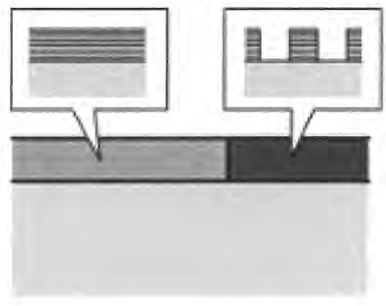

(b)

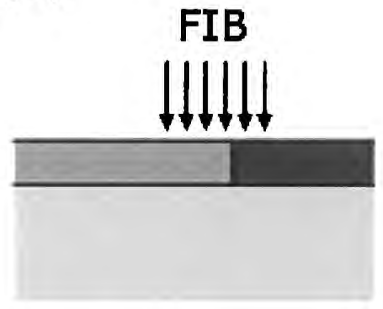

(c)

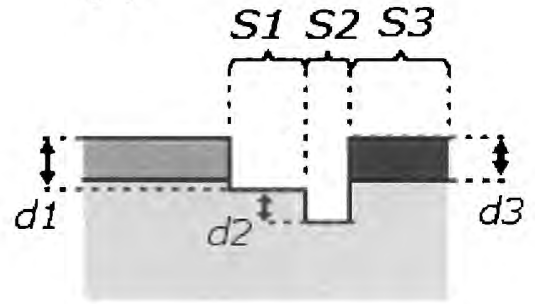

Fig. 5. Additional fabrication process for interferometric observation. (a) Left side, $\mathrm{Al} / \mathrm{SiO}_{2}$ multi layer; right side, fishnet MMs; (b)FIB milling around adjacent region. (c) Side view image of the sample for interferometric observation.

Figure 6(a) represents the interferometric image of the fishnet structure obtained using the interferometric microscope with the incident light of $1,530 \mathrm{~nm}$ in wavelength. In the $150 \mu \mathrm{m}$ square region, the structure shown in Fig. 6(b) was placed, although the holes cannot be observed because their radii are smaller than the diffraction limit. When observing with this system, the interferometric fringes are observed over the ordinary optical microscopic image of the structure.

The interferometric fringes consist of bright bands and dark bands, covering entire sight of the microscope. Each of the bright fringes corresponds to an interference of each order. The distance of two adjacent bright fringes represents the phase difference of $2 \pi$. The fringe displacement between the circular hole region and the square hole region represents the phase difference between them. The phase difference, $\Delta \delta$, which is analyzed from interferometric fringe, is expressed as:

$$
\Delta \delta=2 \pi \Delta m+2 n \pi \quad(n=0,1,2 \cdots)
$$

where $\Delta \mathrm{m}$ is the ratio of the fringe displacement and the distance between adjacent fringes. When fringe displacement is drastically chanced, it means the sample has a sharp border or sharp refractive index change; we cannot attribute the fringe continuity. Therefore above equation has an uncertainty of $2 n \pi$. Under the condition that refractive indices difference $\Delta \mathrm{n}$ are known, above equation is expressed as:

$\Delta \delta=2 \pi \Delta n L / \lambda$ 
where $\mathrm{L}$ is a thickness of the sample, and $\lambda$ is a free-space wavelength. Since interferometric fringes are sensitive against vibration, absolute position of fringes might totally shit. However, the fringe displacement is maintained in any vibration. Therefore we can read the phase difference from this figure

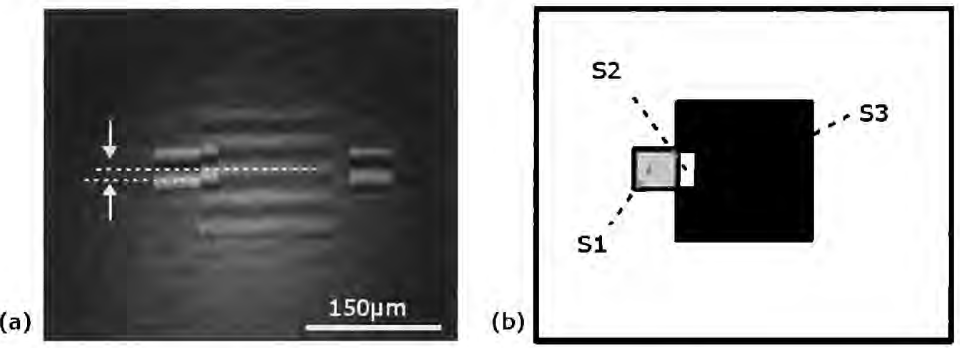

Fig. 6 (a) Interferometric image. Arrow indicates the fringe displacement between S1 and S3. From this fringe displacement and the distance between adjacent fringes, we can determine the phase difference between S1 and S3.

(b) Explanation drawing of (a)

From this measurement, the phase shift of the circular hole and the square hole fishnet were about 110 degrees and 85 degrees, respectively, within a tunable wavelength from $1,470 \mathrm{~nm}$ to $1.545 \mathrm{~nm}$. These values were equivalent to a refractive-index-change of 0.8 and 0.6 , respectively. From these results, it can be said that high dispersivity can be obtained from fishnet structures. The value of the dispersivity can be controlled by the shape of the hole.

\section{CONCLUSION}

We experimentally revealed the controllability of transmission phase of fishnet structure by using the wevelength tunable interferometric microscope. Transmission phases were controlled by a small change of nano-hole's shape. The microscope directly reveals the phase difference between two adjacent regions. Phase differences between the fishnet and the free-space were measured. From this measurement, the phase shift of the circular hole and the square hole fishnet were about 110 degrees and 85 degrees, respectively, within a tunable wavelength from $1,470 \mathrm{~nm}$ to $1,545 \mathrm{~nm}$. These values were equivalent to a refractive-index-change of 0.8 and 0.6 , respectively. From these results, fishnet structures indicate high dispersivity within target wavelengths. Thus, fishnet structures with different hole shapes have a potential to be an element which can control the transmission phase of light.

\section{ACKNOWLEDGEMENT}

This work was partly supported by the New Energy and Industrial Technology Development Organization (NEDO) program.

\section{REFERENCES}

[1] T. W. Ebbesen, H. J. Lezec, H. F. Ghaemi, T. Thio, and P. A. Wolff, "Extraordinary optical transmission through sub-wavelength hole arrays", Nature, 391, 667-669(1998)

[2] F. J. Garcia-Vidal, L. Martin-Moreno, T. W. Ebbesen, L. Kuipers, "Light passing through subwavelength apertures", Rev. of Mod. Phys, 82, 729-787(2010)

[3] K. Busch, G. von Freymann, S. Linden, S. F. Mingaleev, L. Tkeshelashvili, and M. Wegener, "Periodic nanostructures for photonics", Phys. Rep. 444, 101-202(2007)

[4] D. Inoue, A. Miura, T. Nomura, H. Fujikawa, K. Sato, N. Ikeda, D. Tsuya, Y. Sugimoto, and Y. Koide, "Polarization independent visible color filter comprising an aluminum film with surface-plasmon enhanced transmission through a subwavelength array of holes", Appl. Phys. Lett., 98, 093113(2011) 
[5] J. Valentine, S. Zhang, T. Zentgraf, E.Ulin-Avila, D. A. Genov, G. Bartal, and Z. Zhang, "Three-dimensional optical metamaterial with a negative refractive index," Nature, 455, 376-380(2008)

[6] G. Dolling, C. Enkrich, M. Wegener, C. M. Soukoulis, and S. Linden, "Simultaneous Negative Phase and Group Velocity of Light in a Metamaterial," Science, 312, 892-894(2006)

[7] G. Dolling, M. Wegener, C. M. Soukoulis, and S. Linden, "Design-related losses of double-fishnet negativeindex photonic metamaterials", Opt. Exp. 15, 11536-11541(2007)

[8] D. Fattal, J. Li, Z. Peng, M. Fiorentino, and R. G. Beausoleil, "Flat dielectric grating reflectors with focusing abilities", Nature Photon., 4, 466-470(2010)

[9] L. Verslegers, P. B. Catrysse, Z. Yu, J. S. White, E. S. Barmard, M. L. Brongersma, and S. Fan, "Planar Lenses Based on Nanoscale Slit Arrays in a Metallic Film", Nano Lett., 9, 235-238(2009)

[10] G. Dolling, C. Enkrich, M. Wegener, C. M. Soukoulis, and S. Linden, "Low-loss negative-index metamaterial at telecommunication wavelengths", Opt. Lett., 31, 1800-1802(2006)

[11]Z. Ku, and S. R. J. Brueck, "Experimental demonstration of sidewall angle induced bianisotropy in multiple layer negative index metamaterials", Appl. Phys. Lett., 94, 153107(2009)

[12]Max Born and Emil Wolf, "Principles of Optics," 7th edition, fourth printing, (Cambridge University Press, 2006), Chap. 7.

[13]M. B. Sinclair, M. P. de Boer, and A. D. Corwin, "Long-working-distance incoherent-light interference microscope", Appl. Opt. 44, 7714-7721(2005)

[14]Amnon Yariv and Pochi Yeh, "Photonics", Optical Electronics in Modern Communications, Sixth Edition, (Oxford University Press, 2007), Chap. 2. 